\title{
Depressão e Ansiedade de Pessoas Vivendo com HIV
}

\author{
Ingrid Bergmam do Nascimento Silva, ${ }^{1}$ Milenna Azevedo Minhaqui Ferreira, ${ }^{2}$ \\ Anna Cláudia Freire de Araújo Patrício, ${ }^{3}$ Brenda Feitosa Lopes Rodrigues, ${ }^{3}$ \\ Maria Hellena Ferreira Brasil, ${ }^{4}$ João Agnaldo Nascimento, ${ }^{3}$ \\ Richardson Augusto Rosendo da Silva ${ }^{5}$
}

\section{RESUMO}

Objetivo: Analisar a presença de depressão e ansiedade de pessoas vivendo com HIV bem como a associação da depressão com a ansiedade. Método: Estudo transversal, quantitativo, realizado entre janeiro e julho do ano de 2019. Participaram cem pessoas vivendo com HIV que recebiam assistência ambulatorial em Hospital para Doenças Infectocontagiosas no Nordeste, Brasil. Utilizaram-se questionário sociodemográfico, Escala de Depressão de Hamilton e Escala de Ansiedade de Beck. Houve análise estatística descritiva e inferencial com testes de Kruskl Wallis e teste $t$, considerando significativo quando $p \leq 0,05$. Resultados: Identificou-se que $64 \%$ eram homens, $70 \%$ heterossexual, 50\% possuíam entre 20 e 30 anos, $90 \%$ contraíram HIV por contato sexual, 42\% apresentaram depressão e $52 \%$ ansiedade mínima. Evidenciou-se influência da depressão sobre ansiedade ( $p=0,041)$, bem como associação entre os escores de depressão e ansiedade $(p=0,001)$. Conclusão: Comprovou-se a hipótese de que a depressão influencia a ansiedade de pessoas com HIV.

Palavras-chave: Depressão; ansiedade; HIV; Síndrome de Imunodeficiência Adquirida; saúde mental.

\section{DEPRESSION AND ANXIETY OF PEOPLE LIVING WITH HIV}

\section{ABSTRACT}

Objective: To analyze the presence of depression and anxiety of people living with HIV, as well as the association of depression on anxiety. Method: Cross-sectional, quantitative study, conducted between january and july of the year 2019. The participants were 100 people living with HIV who received outpatient care at a Hospital for infectious diseases, northeast, Brazil. A socio-demographic questionnaire, Hamilton Depression Scale and Beck anxiety scale were used. There was descriptive and inferential statistical analysis with KRUSKL Wallis tests and T test, considering significant when $p \leq 0.05$. Results: It was identified that $64 \%$ were men, $70 \%$ heterosexual, $50 \%$ had between 20 and 30 years and $90 \%$ contracted HIV by sexual contact, $42 \%$ had depression and $52 \%$ had minimal anxiety. The influence of depression on anxiety $(p=0,041)$ was evidenced, as well as an association between depression and anxiety scores $(p=0.001)$. Conclusion: The hypothesis that depression influences the anxiety of people with HIV was confirmed.

Keywords: Depression; anxiety; HIV; Acquired Immunodeficiency Syndrome; mental health.

RECEBIDO EM: 16/9/2019

MODIFICAÇÕES SOLICITADAS EM: 27/10/2019

ACEITO EM: 10/3/2021

\footnotetext{
1 Universidade Federal da Paraíba. João Pessoa/PB, Brasil.

2 Autora correspondente. Universidade Federal da Paraíba - Campus I. Lot. Cidade Universitária. João Pessoa/PB, Brasil. CEP 58051-900. http://lattes.cnpq.br/2076421707441687.https://orcid.org/0000-0002-9151-9902. milenna_minhaqui@ hotmail.com

${ }^{3}$ Universidade Federal de Uberlândia. Uberlândia/MG, Brasil.

${ }^{4}$ Universidade Federal da Paraíba. João Pessoa/PB, Brasil.

${ }^{5}$ Universidade Federal do Rio Grande do Norte. Natal/RN, Brasil.
} 


\section{INTRODUÇÃO}

Os avanços terapêuticos e a intensa divulgação voltada para a prevenção da infecção pelo HIV são significativos, entretanto a doença ainda é considerada um problema de saúde pública de ordem mundial ${ }^{1}$. No mundo até o ano 2017, o HIV atingia 36,9 milhões de pessoas ${ }^{2}$ e, no Brasil, entre 1980 e junho de 2018, foram notificados 982.129 casos de aids ${ }^{3}$.

A infecção pelo HIV pode constituir-se como um fator etiológico para o desenvolvimento de perturbações psiquiátricas ou para a exacerbação de condições preexistentes, como a depressão ${ }^{4}$. Devido às consequências sociais adversas decorrentes das crenças e estigmas, o HIV está entre as doenças que não só afetam os aspectos físicos, mas também condições psicológicas e mentais das pessoas diagnosticadas ${ }^{5}$.

A depressão em pessoas com HIV pode favorecer a progressão da doença, acelerando o declínio da função imune, com diminuição significativa da contagem de linfócitos T CD4+, aumentando a taxa de mortalidade ${ }^{4,6}$.

Um estudo realizado em Sidney/Austrália com 264 pessoas com HIV, revelou que $54 \%$ apresentavam transtornos psíquicos, sendo mais predominantes a ansiedade e a depressão. Testes estatísticos revelaram significância estatística entre estas variáveis e a hospitalização e a baixa adesão à terapia antirretroviral $(\text { TARV })^{7}$.

Após a aplicação da Escala Hospitalar de Ansiedade e Depressão (HADS) e a verificação dos dados sociodemográficos, uma análise realizada em um Complexo Hospitalar em Brasília/Brasil trouxe como principal achado a prevalência de depressão e ansiedade em indivíduos que acreditam que o HIV promove uma grande ameaça à sua perspectiva de vida. Além disto, as pessoas com HIV que apresentaram distúrbios psicológicos mostraram sintomas mais graves da doença ${ }^{8}$.

O transtorno depressivo pode ser desencadeado devido à inexistência de cura para o HIV, pelo sentimento de falta de controle sobre o futuro, pelos limites impostos pela doença, vivência ou medo de preconceito e recriminação ${ }^{9}$. Em razão disso, muitas pessoas com HIV adotam condutas de isolamento em consequência da rejeição, enfrentando dificuldades em iniciar novos relacionamentos afetivos, sociais e sexuais. Em consequência, muitos sofrem redução da autoestima e perda da identidade psicológica e social, o que pode desencadear sofrimento psíquico, como a depressão $0^{10}$.

Ressalta-se que a depressão e a ansiedade encontram-se fortemente relacionadas à ideação suicida, pois encontram neste ato a melhor forma de libertar-se do sofrimento físico e emocional, discriminação e isolamento ${ }^{11}$.

A ansiedade caracteriza-se, de modo geral, por preocupação excessiva, agitação psicomotora, isolamento social e sintomas físicos. Estes transtornos são mais prevalentes entre pacientes com doenças crônicas quando comparados com sujeitos saudáveis, sendo sua prevalência de $64 \%$ em comparação com $19,9 \%$ na população geral ${ }^{12}$. Estes fatos prejudicam o funcionamento do trabaIho, o âmbito social, a qualidade de vida e a saúde do indivíduo ${ }^{13}$.

Editora Unijuí - Revista Contexto \& Saúde - ISSN 2176-7114 - v. 21, n. 44, out./dez. 2021 
Nesta perspectiva, este estudo torna-se importante para profissionais da saúde, especificamente enfermeiros que trabalham na assistência às pessoas com HIV, com a finalidade de compreender a presença de depressão e ansiedade nestes indivíduos, bem como a associação entre as variáveis, favorecendo um cuidado específico de prevenção e reabilitação.

Desta forma, este trabalho teve como objetivo analisar a presença de depressão e ansiedade de pessoas vivendo com HIV, bem como a associação da depressão sobre a ansiedade.

\section{MÉTODO}

Estudo transversal, descritivo, quantitativo, realizado em um ambulatório de um Hospital referência em Doenças Infectocontagiosas em uma cidade do Nordeste do Brasil, no período entre janeiro e julho de 2019.

O cálculo do tamanho da amostra de pacientes se deu a partir da fórmula para populações finitas, levando em consideração o nível de confiança de $95 \%$ $(Z \infty=1,96)$, o erro amostral de $10 \%$ e o tamanho da população de 4.850 pacientes cadastrados e acompanhados no referido ambulatório, resultando em uma amostra de 95 pacientes, que se optou por finalizar em um total de cem pacientes, os quais foram selecionados por conveniência, de forma consecutiva. As amostras não probabilísticas supõem um processo de seleção informal e possuem, como vantagem, uma cuidadosa e controlada escolha de casos com certas características especificadas na formulação do problema ${ }^{14}$.

Utilizaram-se os seguintes critérios de inclusão: idade igual ou superior a 18 anos, diagnóstico de HIV, estar recebendo atendimento ambulatorial no período da coleta de dados. Excluíram-se todos aqueles que apresentavam condições físicas e cognitivas prejudicadas que impossibilitassem a coleta de todos os itens dos instrumentos.

Os instrumentos de coleta de dados empregados na pesquisa incluíram: questionário sociodemográfico (idade, sexo, modo de infecção/contágio ao HIV, uso de medicação psiquiátrica, orientação sexual), Escala de Depressão de Hamilton ${ }^{15}$ e Escala de Ansiedade de Beck ${ }^{16}$.

A Escala de Depressão de Hamilton é composta por 17 itens; cada item pode variar de zero até dois ou quatro (dependendo do item em questão), com a soma dos escores variando de zero a 52 pontos. Considera-se como gravemente deprimidos escores com mais de 25 pontos, moderadamente deprimidos escores entre 18 e 24 pontos, e depressão leve escores entre 7 e 17 pontos. Esses itens estão relacionados à insônia, ansiedade, humor deprimido, sintomas orgânicos e ideação suicida ${ }^{15}$. A Escala de Ansiedade de Beck inclui 21 questionamentos, com quatro opções de respostas absolutamente não, levemente, moderadamente e gravemente, com pontuação 0, 1, 2, 3, respectivamente. Desta forma, o escore máximo atinge 63 pontos e classifica a ansiedade em diversos níveis: mínimo (zero a 10 pontos); leve (11 a 19 pontos); moderado (20 a 30 pontos); grave (31 a 63 pontos) $)^{16}$.

Editora Unijuí - Revista Contexto \& Saúde - ISSN 2176-7114 - v. 21, n. 44, out./dez. 2021 
Os instrumentos de coleta de dados foram empregados por enfermeira/ pesquisadora/professora e graduandos em enfermagem treinados previamente por um minicurso com duração de seis horas. A abordagem aos usuários ocorreu na sala de espera do ambulatório, sendo a coleta de dados realizada em um espaço mais reservado com duração, em média, de 40 minutos para cada voluntário.

Os dados foram expressos por meio de estatística descritiva com média, desvio padrão da média, frequência absoluta e relativa. Aplicou-se testes considerando significativo quando $p \leq 0,05$. Para verificar se a classificação da depressão (presente ou ausente) está associada à classificação da ansiedade (mínima, leve, moderada, grave), foi aplicado o teste Kruskal Wallis, e para testar a relação entre os escores das variáveis depressão e ansiedade empregou-se o teste t.

Esta pesquisa foi autorizada pelo local de coleta de dados, aprovada pelo Comitê de Ética em Pesquisa conforme parecer 2.269.394. Foram entregues duas vias do Termo de Consentimento Livre e Esclarecido, sendo uma via devolvida assinada ao pesquisador, atendendo, assim, os critérios estabelecidos na Resolução n 466/2012 do Conselho Nacional de Saúde, que diz respeito às Diretrizes e Normas Regulamentadoras de Pesquisa Envolvendo Seres Humanos ${ }^{17}$.

\section{RESULTADOS}

A Tabela 1 demonstra a caracterização das cem pessoas diagnosticadas com HIV que participaram da pesquisa

Tabela 1 - Caracterização de pessoas vivendo com HIV ( $N=100)$, Nordeste, Brasil, 2019

\begin{tabular}{|c|c|c|}
\hline VARIÁVEIS & $\mathbf{n}$ & $\%$ \\
\hline \multicolumn{3}{|l|}{ Sexo } \\
\hline Masculino & 64 & $64 \%$ \\
\hline Feminino & 36 & $36 \%$ \\
\hline \multicolumn{3}{|l|}{ Idade (anos) } \\
\hline 20 a 30 & 50 & $50 \%$ \\
\hline 31 a 41 & 20 & $20 \%$ \\
\hline 42 a 52 & 15 & $15 \%$ \\
\hline 53 a 63 & 10 & $10 \%$ \\
\hline Maior que 63 anos & 5 & $5 \%$ \\
\hline \multicolumn{3}{|l|}{ Orientação sexual } \\
\hline Heterossexual & 70 & $70 \%$ \\
\hline Homossexual & 30 & $30 \%$ \\
\hline
\end{tabular}

Fonte: Dados da pesquisa, 2019.

No que se refere ao modo de infecção/contágio do HIV, 90\% revelaram ter sido sexual, $5 \%$ com compartilhamento de seringas e $5 \%$ informaram não saber. A Tabela 2 ressalta informações quanto à depressão e ansiedade dos participantes do estudo. 
Tabela 2 - Escore dos questionários de depressão e ansiedade de pessoas vivendo com HIV ( $\mathrm{N}=100)$, Nordeste, Brasil, 2019

\begin{tabular}{lccc}
\hline VARIÁVEIS & \multicolumn{3}{c}{ ESTATÍSTICA } \\
\hline & Média \pm Desvio Padrão & Máximo & Mínimo \\
\cline { 2 - 4 } Escore Depressão & $9,35 \pm 3,27$ & 28 & 5 \\
Escore Ansiedade & $8,56 \pm 2,31$ & 26 & 4 \\
\hline
\end{tabular}

Fonte: Dados da pesquisa, 2019.

Quanto à prevalência de depressão e ansiedade, a Tabela 3 demonstra-as com suas respectivas classificações.

Tabela 3 - Prevalência de depressão e ansiedade de pessoas vivendo com HIV ( $N=100)$, Nordeste, Brasil, 2019

\begin{tabular}{|c|c|c|}
\hline VARIÁVEIS & $\mathbf{N}$ & $\%$ \\
\hline \multicolumn{3}{|l|}{ Depressão } \\
\hline Presente & 42 & $42 \%$ \\
\hline Ausente & 58 & $58 \%$ \\
\hline \multicolumn{3}{|l|}{ Ansiedade } \\
\hline Mínima & 52 & $52 \%$ \\
\hline Leve & 29 & $29 \%$ \\
\hline Moderada & 14 & $14 \%$ \\
\hline Grave & 5 & 5 \\
\hline
\end{tabular}

Fonte: Dados da pesquisa, 2019.

A Tabela 4 demonstra a associação entre as variáveis depressão e ansiedade, com valor $p$ significativo.

Tabela 4 - Associação entre depressão e ansiedade de pessoas vivendo com HIV (N=100), Nordeste, Brasil, 2019

\begin{tabular}{lccc}
\hline \multicolumn{1}{c}{ VARIÁVEIS } & TESTE ESTATíSTICO & VALOR P \\
\hline $\begin{array}{l}\text { A classificação da depressão (presente } \\
\text { ausente) está associada à classificação }\end{array}$ & da & & 0,041 \\
$\begin{array}{l}\text { ansiedade (mínima, leve, moderada, grave). } \\
\text { Relação entre os escores das variáveis depressão }\end{array}$ & Teste t & 0,001 \\
e ansiedade. & & \\
\hline
\end{tabular}

Fonte: Dados da pesquisa, 2019.

\section{DISCUSSÃO}

No que diz respeito à maior prevalência do sexo masculino, essa informação é coerente com os dados de notificação no país ${ }^{3}$. Um estudo realizado com 120 pessoas em um Hospital Referência em Doenças Infectocontagiosas no Nordeste Brasileiro, demonstrou que aproximadamente $65 \%$ dos indivíduos eram do sexo masculino(18), assim como a pesquisa realizada em 116 municípios no Estado do Paraná, no Sul do Brasil, com 5.030 participantes, que também revelou uma porcentagem do sexo masculino equivalente a $60 \%{ }^{19}$. A pesquisa destaca que estes dados podem ser justificados pelo fato de que os homens sentem menos 
necessidade de utilizar o preservativo, e ainda leva em consideração uma maior possibilidade de infecção pelas mulheres ${ }^{20}$.

Apesar da prevalência do HIV em indivíduos do sexo masculino, observa-se um aumento significativo do HIV entre mulheres. O HIV perpassa por um processo de "feminização", no qual mulheres em idade fértil, grávidas e crianças apresentam infecção com maior frequência. A mortalidade materna, considerada uma grande violação dos direitos humanos, possui como um fator de risco a aids, geralmente nas mulheres sintomáticas, devido a problemas recorrentes da imunidade fragilizada ${ }^{21}$.

Os achados deste estudo, nos quais o maior número de pessoas que vivem com HIV são heterossexuais, demonstram uma alteração do cenário quanto ao início da epidemia, quando existiu uma alta taxa de incidência do HIV em homens homossexuais ${ }^{22}$, sugerindo uma mudança no perfil quanto à orientação sexual, evidenciando, assim, que o HIV pode se adentrar em realidades e contextos diversos.

Dentre os entrevistados nesta pesquisa, a maior parte encontrava-se na faixa etária de 20 a 30 anos. Esses dados são distintos de um estudo realizado também no nordeste brasileiro, onde a maior parte da amostra era composta por pessoas com idade superior a 41 anos, com prevalência de 55,2\% ${ }^{23}$.

As principais formas de transmissão do HIV ocorrem pelo contato sexual (vaginal, oral ou anal) desprotegido, compartilhamento de seringas e de forma vertical (da mãe para o filho na hora do parto) ${ }^{24}$. O presente estudo demonstra o maior índice de infecção por transmissão via sexual, dado que corrobora um estudo realizado em Montevideo (Uruguai), detectando que mais de $80 \%$ dos entrevistados foram infectados por via sexual, seguido de $10 \%$ sanguínea e 1,2\% por transmissão vertical ${ }^{25}$.

Esta pesquisa mostrou que os entrevistados apresentaram escore na Escala de Depressão de Hamilton como depressão presente. Dados de uma outra pesquisa vão ao encontro do presente estudo, pois a maioria dos entrevistados apresentou sentimentos depressivos. Apesar do avanço do tratamento antirretroviral e do aumento da expectativa de vida relacionada ao HIV, a incidência desses sintomas persistem, principalmente em pessoas do sexo feminino; grande parte é consequência do isolamento do convívio social e familiar ao invés do enfrentamento ativo ${ }^{26}$.

Um estudo demonstra que o diagnóstico de enfermagem, intitulado angústia espiritual, pode ser utilizado com frequência nos indivíduos com HIV com as características definidoras: culpa, falta de autoperdão, falta de finalidade e significado de vida, e quando expressam os sentimentos vivenciados por estas pessoas ${ }^{27}$.

As pessoas que vivem com HIV, apesar de, muitas vezes, apresentarem-se assintomáticas, podem desenvolver transtornos na esfera psicossocial, e o mais comum deles é a ansiedade. A carência social causa impacto na qualidade de vida e as pessoas tendem a ficar ansiosas na busca da recuperação do espaço social, além do medo quanto ao futuro ${ }^{28}$. 
É possível identificar, na literatura científica, que a depressão e a ansiedade nas pessoas que vivem com HIV geralmente são causadas por sentimento de culpa, medo da morte, perda de peso e isolamento social. Devido a isso, a equipe multiprofissional deve possuir papel ativo na assistência a essas pessoas, influenciando positivamente na adesão ao tratamento medicamentoso, realizando atividades com a rede de apoio e fortalecendo as práticas de atenção ao biopsicossocial do indivíduo ${ }^{29}$.

Após a aplicação da Escala de Ansiedade de Beck, observou-se que metade dos participantes apresentou ansiedade mínima. Outro estudo, que utilizou o mesmo instrumento, obteve resultados semelhantes a este. Além disto, testes estatísticos comprovaram a associação da ansiedade com o grau de estresse percebido $^{29}$. O transtorno de ansiedade encontra-se presente no cotidiano de pessoas que vivem com HIV, representado por $61,6 \%$ em estudo multicêntrico realizado nos EUA com 1.027 indivíduos em contrapartida à prevalência de $37 \%$ na população geral, demonstrando que o HIV contribui para ansiedade ${ }^{30}$.

Pessoas diagnosticadas com HIV podem apresentar fatores associados que contribuem para a alta prevalência de depressão e ansiedade, como isolamento, apoio social prejudicado, medo de revelar o diagnóstico, início tardio da Tarv, estresse. Este último fator está presente durante toda a vida de pessoas com HIV, sendo necessário moldá-lo de modo que, ao longo do tempo, estas pessoas saibam lidar com esta infecção. Para isto, tem-se como estratégias reduzir o comprometimento da saúde mental por meio da triagem, encaminhamento precoce para serviço de saúde mental, acompanhamento realizado por profissionais capacitados e apoio social ${ }^{31}$.

Analisar os níveis de ansiedade e depressão nas pessoas que vivem com HIV é de grande significância para os profissionais de saúde e para a sociedade, pois o estigma criado sobre essas pessoas, o preconceito e a exclusão estão presentes, aumentando a incidência de distúrbios psicossociais. A equipe multiprofissional deve ser responsável por auxiliar na criação de políticas que reinsiram essas pessoas na sociedade, e, além disso, deve oferecer acompanhamento psicológico e tratamento de forma humanizada.

As limitações deste estudo estão relacionadas aos instrumentos utilizados, os quais não permitem descrever as causas da ansiedade e da depressão dos entrevistados. Torna-se relevante, entretanto, por se tratar de uma temática atual, possuir uma amostra significativa e ter apresentado o resultado de testes estatísticos associando às variáveis.

\section{CONCLUSÃO}

Esta pesquisa detectou que $42 \%$ da amostra apresentam-se depressivos, $52 \%$ possuem ansiedade mínima, e, além disso, os escores entre as duas variáveis apresentam associação significativamente estatística, comprovando a hipótese de que a depressão influencia a ansiedade de pessoas com HIV.

Diante do exposto, torna-se essencial proporcionar assistência multiprofisisonal capacitada para evitar e recuperar sentimentos que possam gerar depressão e ansiedade em pessoas com HIV, pois estes são considerados fatores

Editora Unijuí - Revista Contexto \& Saúde - ISSN 2176-7114 - v. 21, n. 44, out./dez. 2021 
importantes na adesão terapêutica, surgimento de doenças oportunistas e alterações na imunidade.

Desta forma, para prevenir ou minimizar a depressão e a ansiedade, torna-se essencial instituir ações que envolvam além da oferta de medicamentos, como prática de atividade física, valorização do ser, espiritualidade, humanização na oferta de cuidados, eliminação de julgamentos, atividades laborais que permitam ao indivíduo sentir-se útil. Neste meio destaca-se a ação de enfermeiros que atuam nesta área e estão vinculados às Estratégias de Saúde da Família, que podem proporcionar acompanhamento mais próximo do cotidiano destas pessoas com HIV por intermédio da criação de grupos de apoio que permitam conversação, troca de experiências e melhor entendimento da doença.

Por fim, sugere-se a elaboração de novas pesquisas, de forma longitudinal, sobre esta temática em outras cidades, para permitir o conhecimento dos índices em outras regiões, assim como a utilização de outros instrumentos que analisem os fatores causais da depressão e ansiedade de pessoas vivendo com HIV.

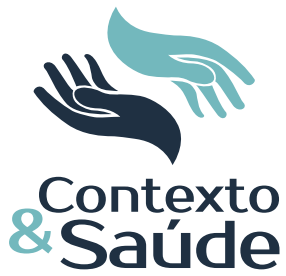

\section{REFERÊNCIAS}

${ }^{1}$ Guimarães MDC, Carneiro M, Abreu DMX, França EB. Mortalidade por HIV/Aids no Brasil, 2000-2015: motivos para preocupação? Rev. Bras. Epidemiol. 2017;20(Suppl 1):182-190. doi: https://doi.org/10.1590/1980-5497201700050015

2 Unaids. Estatísticas Globais Sobre HIV: 2018. Brasília, DF: Unaids; 2018. Disponível em: http://www.unaids.org/sites/default/files/media_asset/unaids-data-2018_en.pdf.

${ }^{3}$ Brasil. Ministério da Saúde. Secretaria de Vigilância em Saúde. Departamento de Vigilância, Prevenção e Controle das Infecções Sexualmente Transmissíveis, do HIV/Aids e das Hepatites Virais. Boletim Epidemiológico HIV/Aids. Brasília, DF: Ministério da Saúde; 2018.

${ }^{4}$ Freitas P, Fernandes A, Morgado P. Depression in HIV-positive patients: the reality of a Portuguese hospital. Sci Med. 2015;25(2):ID20469. doi: https://doi. org/10.15448/1980-6108.2015.2.20469

${ }^{5}$ Dejman M, Ardakani HM, Malekafzali B, Moradi G, Gouya MM, Shushtari ZJ et al. Psychological, Social, and Familial Problems of People Living with HIV/Aids in Iran: a qualitative study. Int J Prev Med. 2015;6(126):1-20. doi: https://doi.org/10.4103/20087802.172540

${ }^{6}$ Leite MA. Depressão, qualidade de vida e adesão ao tratamento antirretroviral em idosos portadores de HIV/Aids. [dissertação]. São Paulo: Programa de Pós-Graduação em Ciências da Coordenadoria de Controle de Doenças da Secretaria de Estado da Saúde de São Paulo; 2016.

${ }^{7}$ Chan DJ, Furner V, Smith DE, Dronavalli M, Bopage RI, Post JJ, et al. Non-AIDS complexity amongst patients living with HIV in Sydney: risk Factors and health outcomes. AIDS Res Ther. 2018;15(6):1-7. doi: https://doi.org/10.1186/s12981-018-0193-z

${ }^{8}$ Nogueira GS, Seidl EMF. Associação entre percepção de doença e ansiedade, depressão e autoeficácia em pessoas com HIV/Aids. Temas Psicol. 2016;24(2):595-608. doi: http://dx.doi.org/10.9788/TP2016.2-12

${ }^{9}$ Coutinho MFC, O’Dwyer G, Frossard V. Tratamento antirretroviral: adesão e a influência da depressão em usuários com HIV/Aids atendidos na atenção primária. Saúde Debate. 2018;42(116):148-161. doi: https://doi.org/10.1590/0103-1104201811612

${ }^{10}$ France NF, Mcdonald SH, Conroy RR, Byrne E, Mallouris C, Hodgson I, Larkan FN. "An unspoken world of unspoken things": a study identifying and exploring core beliefs underlying self-stigma among people living with HIV and Aids in Ireland. Swiss Med Wkly. 
2015;13(145): w14113. doi: https://doi.org/10.4414/smw.2015.14113. eCollection 2015

${ }^{11}$ Wang W, Xiao C, Yao X, Yang Y, Yan H, Li S. Psychosocial health and suicidal ideation among people living with HIV/AIDS: a cross-sectional study in Nanjing, China. Plos one. 2018;13(2):1-17. doi: https://doi.org/10.1371/journal.pone.0192940

12 Silva FS, Oliveira FBM, Mendes CC, Silva G, Santos SO, Pessoa RBC. Preditores associados à qualidade de vida em pessoas vivendo com Hiv/Aids: revisão integrativa. Rev Pre Infec e Saúde. 2015;1(2):53-63. doi: https://doi.org/10.26694/repis.v1i2.3589

${ }^{13}$ Deshmukh NN, Borkar AM, Deshmukh JS. Depression and its associated factors among people living with HIV/AIDS: Can it affect their quality of life? J Family Med Prim Care. 2017;6(3):549-553. https://doi.org/10.4103/2249-4863.222016

${ }^{14}$ Sampieri RH, Collado CF, Lucio MDPB. Metodologia da pesquisa. 5. ed. Porto Alegre: Penso; 2013.

${ }^{15}$ Gallucci Neto J, Campos Júnior MS, Hubner CVK. Escala de Depressão de Hamilton (HAM-D): revisão dos 40 anos de sua utilização. Rev Fac Ciênc Méd. [Internet] 2001 [citado 4 fev. 2021;3(1):10-4. Disponível em: https://revistas.pucsp.br/index.php/RFCMS/ article/view/259/207

${ }^{16}$ Cunha JA. Manual da versão em português das Escalas Beck. São Paulo: Casa do Psicólogo; 2001.

17 Brasil. Conselho Nacional de Saúde. Resolução no 466, de 12 de dezembro de 2012. Aprova diretrizes e normas regulamentadoras de pesquisa envolvendo seres humanos. Diário Oficial da União. Brasília, DF: Ministério da Saúde; 2012.

18 Souza Neto VL, Silva RAR, Rocha CPT, Costa RTS, Nóbrega MML. ICNP ${ }^{\circledR}$ nursing diagnoses for people with acquired immunodeficiency syndrome. Acta Paul. Enferm. 2017 dez.;30(6):573-581. doi: https://doi.org/10.1590/1982-0194201700081

${ }^{19}$ Vogler IH, Alfieri DF, Gianjacomo HDB, Aleida ERD, Reiche EMV. Cascade of care for people living with HIV infection in Southern Brazil: results from a public health network. Cad. Saúde Pública. 2018;34(12):e00009718. doi: http://dx.doi.org/10.1590/ 0102-311x00009718

20 Villela WV, Barbosa RM. Prevenção da transmissão heterosexual do HIV entre muIheres: é possível pensar estratégias sem considerar suas demandas reprodutivas? Rev. Bras. Epidemiol. 2015;18(Suppl 1):131-142. doi: https://doi.org/10.1590/18094503201500050010

${ }^{21}$ Brayner MC, Alves SV. Classification of deaths in women with human immunodeficiency virus/acquired immunodeficiency syndrome in pregnancy and childbirth. Rev. Bras. Epidemiol. 2017;20(3):371-381. doi: https://doi.org/10.1590/1980-5497201700030002

22 Melo EA, Maksud I, Agostini R. Cuidado, HIV/Aids e atenção primária no Brasil: desafio para a atenção no Sistema Único de Saúde? Rev Panam Salud Publica. 2018;42(23):e151. doi: https://doi.org/10.26633/RPSP.2018.151

${ }^{23}$ Silva JG, Morgan DAR, Alchieri JC, Medeiros HF, Knackfuss MI. Pain level associated to socio-demographic and clinical variables in people living with human immunodeficiency virus and acquired immunodeficiency syndrome. Rev. Dor. 2017;18(1):51-58. doi: https://doi.org/10.5935/1806-0013.20170012

${ }^{24}$ Brasil. Secretaria Municipal de Saúde. Subsecretaria de Atenção Primária, Vigilância e Promoção da Saúde. Superintendência de Atenção Primária. Coleção Guia de Referência Rápida. Infecção pelo HIV e Aids: prevenção, diagnóstico e tratamento na atenção primária. Brasília, DF: Ministério da Saúde; 2016.

${ }^{25}$ Alonso SGC, Dalchiele ZA, Rouco JJM, Ferrari FC. Calidad de vida relacionada a la salud en personas con VIH y factores asociados. Rev. Méd. Urug. 2018;34(1):3-41. doi: https://dx.doi.org/10.29193/rmu.34.1.1

${ }^{26}$ Reis RK, Castrighini CC, Melo ES, Jesus GJ, Queiroz AAFL, Gir E. Assessment of somatic and affective-cognitive symptoms of people living with HIV/Aids. Acta Paul. Enferm. 2017;30(1):60-65. doi: https://doi.org/10.1590/1982-0194201700009 
${ }^{27}$ Pinho CM, Gomes ET, Trajano MFC, Cavalcanti ATA, Andrade MS, Valença MP. Impaired religiosity and spiritual distress in people living with HIV/Aids. Rev. Gaúcha Enferm. 2017;38(2):e67712. doi: https://doi.org/10.1590/1983-1447.2017.02.67712

28 Pupulin ART, Herold P, Monteiro MA, Romancini JLH, Pimentel GGA. Efeito de exercícios físicos e de lazer sobre os níveis de cortisol plasmático em pacientes com Aids. Rev. Bras. Ciênc. Esporte. 2016;38(4):328-333. doi: https://doi.org/10.1016/j. rbce.2014.04.001

${ }^{29}$ Calvetti PU, Giovelli GRM, Gauer GJC, Moraes JFD. Níveis de ansiedade, estresse percebido e suporte social em pessoas que vivem com HIV/Aids. Psic.: Teor. e Pesq. 2016;32(4):1-4. doi: https://doi.org/10.1590/0102.3772e324317

${ }^{30}$ Cook JA, Burke-Miller J, Steigman PJ, Schwartz RM, Hessol NA, Milam J et al. Prevalence, comorbidity, and correlates of psychiatric and substance use disorders and associations with HIV Risk Behaviors in a Multisite Cohort of Women Living with HIV. AIDS Behav. 2018;22(10):3141-54. doi: https://doi.org/10.1007/s10461-018-2051-3

${ }^{31}$ Oliveira FBM, Queiroz AAFLN, Sousa AFL, Moura MEB, Reis RK. Sexual orientation and quality of life of people living with HIV/Aids. Rev. Bras. Enferm. 2017;70(5):1004-1010. doi: https://doi.org/10.1590/0034-7167-2016-0420 